\title{
Establishment of BmoR-based biosensor to screen isobutanol overproducer
}

\author{
Huan Yu', Ning Wang ${ }^{1}$, Wenbo Huo ${ }^{1}$, Yuhong Zhang ${ }^{2}$, Wei Zhang ${ }^{2}$, Yu Yang ${ }^{1}$, Zhenya Chen ${ }^{1 *}$ and Yi-Xin Huo ${ }^{1 *}$ (D)
}

\begin{abstract}
Background: Isobutanol, a C4 branched-chain higher alcohol, is regarded as an attractive next-generation transport fuel. Metabolic engineering for efficient isobutanol production has been achieved in many studies. BmoR, an alcoholregulated transcription factor, mediates a $\sigma^{54}$-dependent promoter $P_{b m o}$ of alkane monooxygenase in $n$-alkane metabolism of Thauera butanivorans and displays high sensitivity to C4-C6 linear alcohols and C3-C5 branched-chain alcohols. In this study, to achieve the high-level production of isobutanol, we established a screening system which relied on the combination of BmoR-based biosensor and isobutanol biosynthetic pathway and then employed it to screen isobutanol overproduction strains from an ARTP mutagenesis library.

Results: Firstly, we constructed and verified a GFP-based BmoR- $P_{\text {bmo }}$ device responding to the isobutanol produced by the host. Then, this screening system was employed to select three mutants which exhibited higher GFP/OD 600 values than that of wild type. Significantly, GFP/OD 600 of mutant 10 was $190.7 \pm 4.8$, a 1.4-fold higher value than that of wild type. Correspondingly, the isobutanol titer of that strain was $1597.6 \pm 129.6 \mathrm{mg} / \mathrm{L}$, 2.0-fold higher than the wild type. With the overexpression of upstream pathway genes, the isobutanol production from mutant 10 reached $14.0 \pm 1.0 \mathrm{~g} / \mathrm{L}$ after medium optimization in shake flask. The isobutanol titer reached $56.5 \pm 1.8 \mathrm{~g} / \mathrm{L}$ in a fed-batch production experiment.

Conclusions: This work screened out isobutanol overproduction strains from a mutagenesis library by using a screening system which depended on the combination of BmoR-based biosensor and isobutanol biosynthetic pathway. Optimizing fermentation condition and reinforcing upstream pathway could realize the increase of isobutanol production from the overproducer. Lastly, fed-batch fermentation of the mutant enhanced the isobutanol production to $56.5 \pm 1.8 \mathrm{~g} / \mathrm{L}$.
\end{abstract}

Keywords: BmoR, Biosensor, Screening, Isobutanol, Mutagenesis

\section{Background}

Higher alcohols including $n$-butanol, isobutanol, 2-methyl-1-butanol (2-MB), 3-methyl-1-butanol (3-MB) and 2-phenylethanol have drew much attention as nextgeneration transport fuels because of their higher energy density, lower vapor pressure and lower hygroscopicity when compared with traditional biofuel such as ethanol [1]. Microbial-based metabolic engineering, as an eco-friendly strategy, has been used to produce many

\footnotetext{
*Correspondence: chenzhenya@bit.edu.cn; huoyixin@bit.edu.cn ${ }^{1}$ Key Laboratory of Molecular Medicine and Biotherapy, School of Life Science, Beijing Institute of Technology, No. 5 South Zhongguancun Street, Beijing 100081, China

Full list of author information is available at the end of the article
}

value-added compounds from renewable resources [27]. Microbial production of higher alcohols also has been realized in many engineered hosts $[3,8-10]$. The pathways for the biosynthesis of these alcohols were extended from 2-keto acids, the intermediates in amino acid biosynthetic pathways. Subsequently, 2-keto acids were converted to corresponding alcohols by the sequential catalysis through 2-keto acid decarboxylase (KDC) and alcohol dehydrogenase (ADH) $[11,12]$.

The precursor for isobutanol biosynthesis was 2-ketoisovalerate (2-KIV), an intermediate in L-valine biosynthetic pathway which could be biosynthesized from pyruvate through a three-step enzymatic catalysis by the enzymes AlsS, IlvC and IlvD (Fig. 1). The conversion 
between 2-KIV and L-valine was catalyzed by an endogenous aminotransferase. So far, many microorganisms such as Escherichia coli (E. coli), Bacillus subtilis (B. subtilis), Saccharomyces cerevisiae (S. cerevisiae) and Corynebacterium glutamicum (C. glutamicum) have been engineered to produce isobutanol [8, 13-15]. In these studies, the ketoisovalerate decarboxylase (Kivd) and alcohol dehydrogenase (AdhA) would catalyze 2-KIV to isobutyraldehyde and convert isobutyraldehyde to isobutanol, respectively. Hence, assembling the endogenous 2-KIV biosynthetic pathway and exogenous Kivd and AdhA could realize the biosynthesis of isobutanol from glucose. To broaden the carbon source for higher alcohols production, Huo et al. employed single-cell protein wastes as carbon source and reached $56 \%$ of the theoretical yield [16].

In general, modifying microorganism was an essential step in metabolic engineering. Microbial mutation breeding was a common strategy which included random mutagenesis by nitrosoguanidine (NTG) [17], ultraviolet treatment (UV) [18] or atmospheric and room temperature plasma (ARTP) $[19,20]$. After mutagenesis of the host microorganism, the methods for screening mutant strains that overproduced the target molecules or their relevant precursors were desired. Traditional screening methods depended on high performance liquid chromatography (HPLC) or gas chromatograph (GC), which were time-consuming and limited by high cost and low efficiency. Biosensor, as an emerging tool, could respond to the specific metabolite and then dynamically regulate the whole metabolic pathway, leading to the balance of metabolic flux and the increase of the productivity of desired compound [21-26]. Biosensors were used in many studies to regulate the expressions of fluorescent proteins, which provided the detection signals for highthroughput screening [27-29]. Zhang et al. constructed NCgl0581 biosensor in C. glutamicum to screen out a high-producing L-serine strain that produced $34.78 \mathrm{~g} / \mathrm{L}$ L-serine with a yield of $0.35 \mathrm{~g} / \mathrm{g}$ sucrose, which were 35.9 and $66.7 \%$ higher than those of the parent strain [30]. Liu et al. adopted the L-phenylalanine-specific transcription factor (TyrR) in E. coli to screen out a variant which could yield 1.8-fold higher L-phenylalanine when compared with the parent strain [31].

So far, using a strategy for screening high-level isobutanol production strains from the mutagenesis library has not been accomplished. BmoR, an alcohol-regulated transcription factor in $n$-alkane metabolism of Thauera butanivorans, mediated a $\sigma^{54}$-dependent promoter $P_{b m o}$ of alkane monooxygenase [32]. The alcohol molecules could bind to BmoR (an enhancer-binding protein as hexamer) and the generated combination would remodel $\sigma^{54}$-RNAP holoenzyme $\left(E \sigma^{54}\right)$ closed complex and then activate the transcription initiation of promoter $P_{b m o}$ [33]. Dietrich et al. firstly used BmoR as a biosensor to respond to various alcohols and the

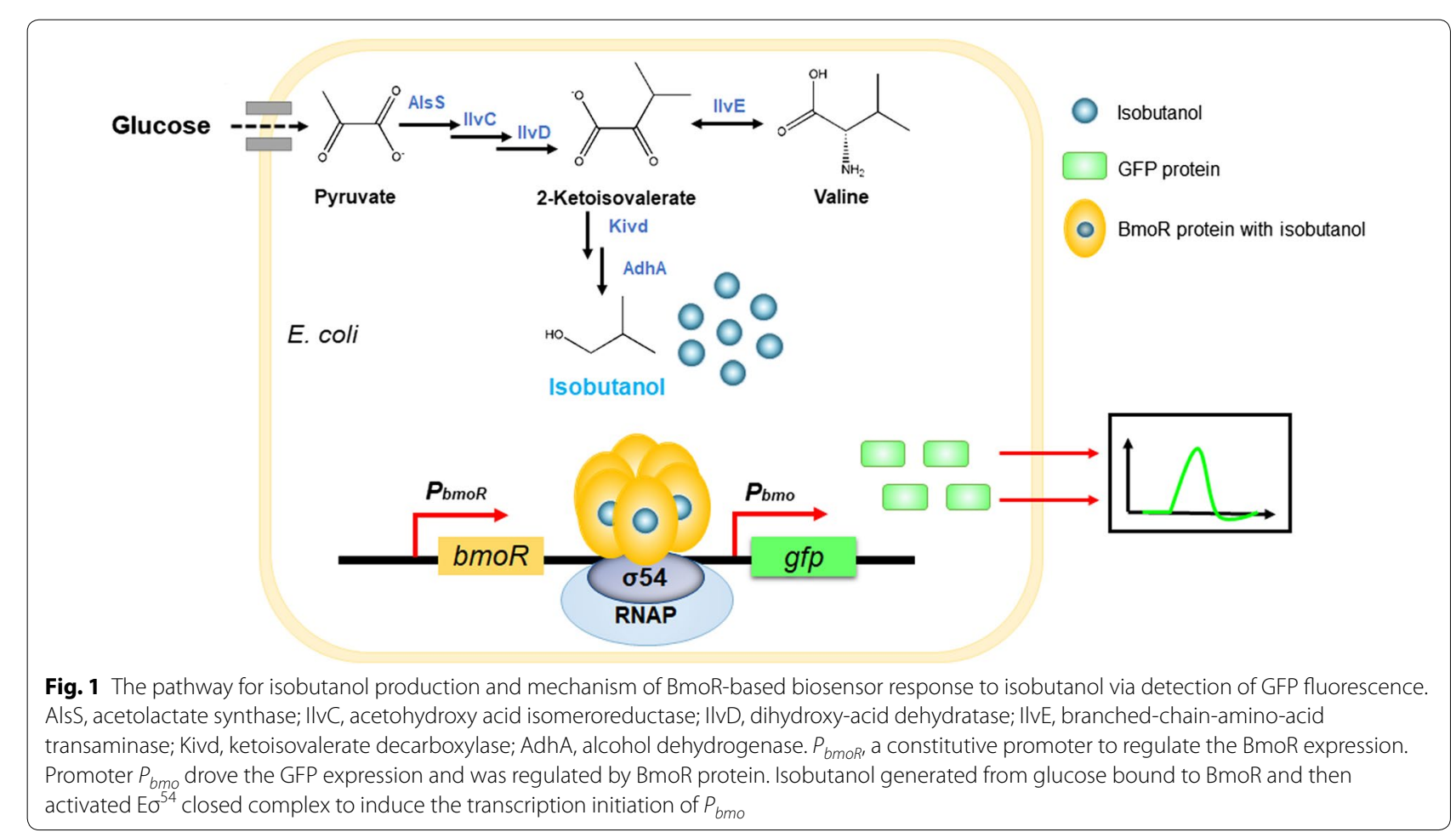


results showed that BmoR exhibited high sensitivity to C4-C6 linear alcohols and C3-C5 branched-chain alcohols, of which $n$-butanol showed the broadest linear range of detection (from $100 \mu \mathrm{M}$ to $40 \mathrm{mM}$ ). Then, BmoR-based biosensor was employed to screen out the strain with the highest conversion rate from 2-oxopentanoate to $n$-butanol in a RBS mutagenesis library [34].

For the purpose of acquiring the high-level isobutanol production strains, it is essential to acquire efficient host microorganisms. In this study, a biosensor-based strategy was designed for screening isobutanol overproduction strains. Isobutanol generated from glucose in hosts or absorbed from environment would bind to BmoR hexamer whose expression was driven by a constitutive promoter. The BmoR with isobutanol binding would activate $\mathrm{E}^{54}$ closed complex and then induce the transcription initiation of promoter $P_{b m o}$. The whole response process was shown in Fig. 1. Firstly, we constructed a GFP-based BmoR- $P_{b m o}$ biosensor system and testified its effectiveness. Then, this biosensor system combining with isobutanol biosynthetic pathway was introduced into the mutagenesis library to screen the isobutanol overproducers. As a result, the mutant which was screened out from the mutagenesis library could produce $14.0 \pm 1.0 \mathrm{~g} / \mathrm{L}$ isobutanol in shake flask and $56.5 \pm 1.8 \mathrm{~g} / \mathrm{L}$ isobutanol in a 3-L bioreactor. This work illustrated that BmoRbased biosensor could respond to intracellular isobutanol and could be used to screen isobutanol overproducers, which has the potential to be engineered to overproduce L-valine since the biosynthesis pathways of isobutanol and L-valine shared the same precursor 2-KIV.

\section{Results and discussion}

\section{Characterization of BmoR- $P_{b m o}$ biosensor via feeding isobutanol}

To verify the response of the BmoR-based biosensor to alcohols, $g f p$ was chosen as a reporter gene under the control of promoter $P_{b m o}$. E. coli BW25113 (F') harboring plasmid pYH1 (YH1) was used for subsequent experiments. To conduct the experiments, $50 \mu \mathrm{L}$ pre-culture of strain $\mathrm{YH} 1$ and $950 \mu \mathrm{L}$ fresh LB medium supplemented with different final concentrations $(0,0.01,0.1,1,10,20$, 40,50 or $100 \mathrm{mM}$ ) of $n$-butanol, isobutanol and $3-\mathrm{MB}$, respectively, were added into in 96 -deep-well plates and the cultures were then left at $30{ }^{\circ} \mathrm{C}$ incubator for $16 \mathrm{~h}$. Then, microplate reader was used to detect the GFP fluorescence and $\mathrm{OD}_{600}$ values. The fluorescence microscope (Nikon model Eclipse Ni-U) was used to observe fluorescence distribution of the cells. As the results shown in Fig. 2a, the fluorescence distributed evenly in the respective cell and all the cells had the similar fluorescence intensity when feeding $50 \mathrm{mM}$ isobutanol to the culture. The response which represented as GFP/OD 600 values were shown in Fig. $2 \mathrm{~b}$. The response of this biosensor was improved along with the increase of the concentration of alcohols. The response of this biosensor to $n$-butanol was higher than the response of other two alcohols whatever the concentration of the alcohols was. The response of the biosensor in the presence of isobutanol or 3-MB feeding was weak if the concentations of the alcohols were lower than $1 \mathrm{mM}$. When the concentration of isobutanol was increased to $10 \mathrm{mM}$, the GFP/ $\mathrm{OD}_{600}$ value was $168.6 \pm 4.13$, a similar value to $3-\mathrm{MB}$ but was 1.4-fold lower when compared with $n$-butanol.
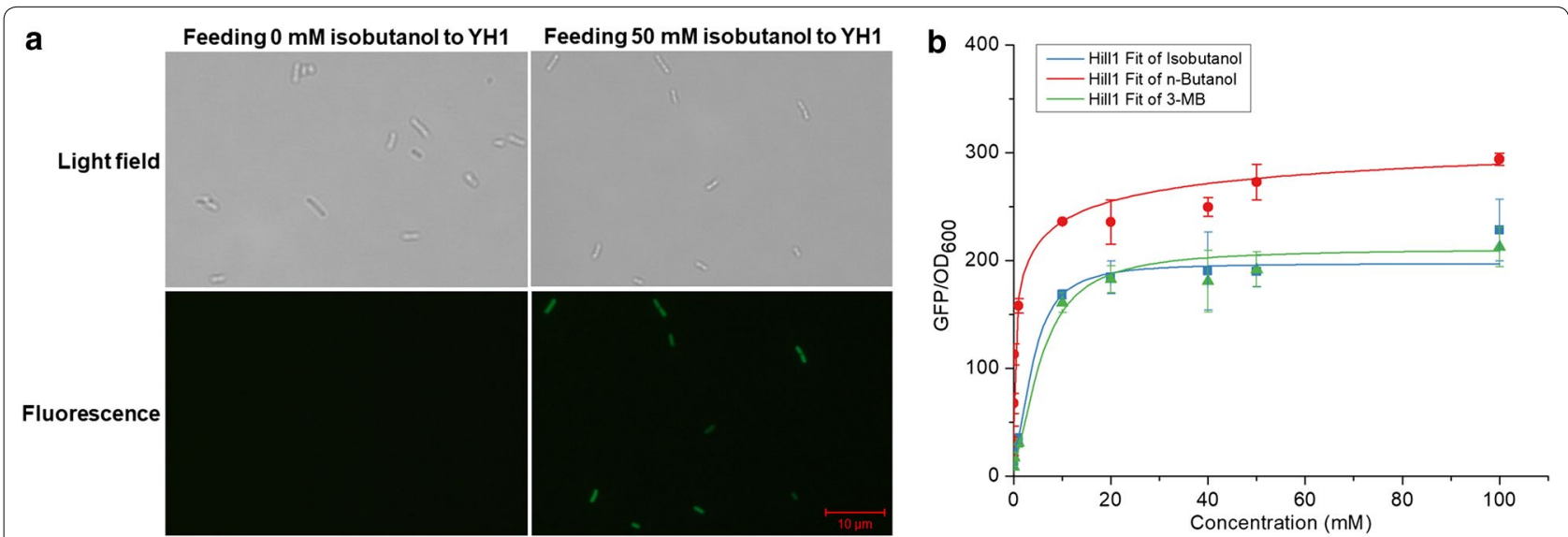

Fig. 2 Fluorescence distribution of the cells and the response of BmoR-based biosensor to different alcohols which were fed into the culture. Strain YH1 (BW25113 (F') harboring plasmid pYH1) was used for fluorescence detection. For a, the fluorescence microscope (Nikon model Eclipse $\mathrm{Ni}-U$ ) was used to observe fluorescence distribution of the cells. Fluorescence was not observed when feeding $0 \mathrm{mM}$ isobutanol to the culture and was observed when feeding $50 \mathrm{mM}$ isobutanol. For $\mathbf{b}$, the response values of this biosensor towards isobutanol, $n$-butanol and 3-MB which were represented by blue, red and green lines, respectively 
The GFP/OD ${ }_{600}$ towards isobutanol reached $228.2 \pm 28.5$ when the isobutanol concentration reached $100 \mathrm{mM}$. Further enhancing the isobutanol concentration would inhibit the cell growth and then resulted in the decrease of response, similar as the other two alcohols. Table 1 displayed the $K_{\mathrm{m}}$ of this biosensor towards these three alcohols. This biosensor has a $K_{\mathrm{m}}$ of $4.2 \pm 0.3 \mathrm{mM}$ towards isobutanol which was close to 3-MB and 1.8-fold higher than $n$-butanol. Besides, blue-white screening experiment was conducted to confirm the response of BmoRbased biosensor towards alcohols which was fed to the culture. The single colonies of E. coli BW25113 $\left(\mathrm{F}^{\prime}\right)$ harboring pYH2 (YH2) which spread on the LB plate with different concontrations of isobutanol and $100 \mu \mathrm{g} / \mathrm{mL}$

Table 1 GFP/OD 600 max and $K_{m}$ values of BmoR-based biosensor towards isobutanol, $n$-butanol and 3-MB

\begin{tabular}{lll}
\hline Compound & $\begin{array}{l}\text { Dynamic range (GFP/ } \\
\text { OD }_{\mathbf{6 0 0 m a x}}\end{array}$ & $\boldsymbol{K}_{\mathbf{m}}(\mathbf{m M})$ \\
\hline Isobutanol & $197.8 \pm 51.2$ & $4.2 \pm 0.3$ \\
n-Butanol & $336.8 \pm 137$ & $2.3 \pm 1.1$ \\
3-MB & $200.7 \pm 7.5$ & $4.6 \pm 1.1$ \\
\hline
\end{tabular}

X-gal adding were not able to turn blue (data not shown), probably due to the evaporation of isobutanol. Taken together, this biosensor could respond to $n$-butanol, isobutanol and 3-MB and had significant response when the concentration of alcohols exceeded $1 \mathrm{mM}$.

\section{Optimizing the copy number of BmoR- $P_{b m o}$ device in the isobutanol-producing strains}

The blue-white screening experiment was performed on the strains YHS1, YHS2 and YHS3 which have the BmoR-based biosensor and the ability of producing isobutanol. As shown in Fig. 3, YHS1 containing BmoR$P_{b m o}$ device in a high-copy-number plasmid turned blue on the X-gal plates without IPTG induction, illustrating that high-copy-number plasmid were not suitable for this biosensor construction due to the leaking expression of $P_{L} l a c O_{1}$ promoter. On the contrary, the colonies of strain YHS3 which containing BmoR- $P_{b m o}$ device in a low-copy-number plasmid were unable to turn blue in spite of IPTG inducing. Fortunately, the colonies of strain YHS2 containing BmoR- $P_{b m o}$ device in a medium-copynumber plasmid could only turn blue in the presence of IPTG induction, suggesting the medium-copy-number plasmid was optimal for constructing BmoR- $P_{b m o}$ device.
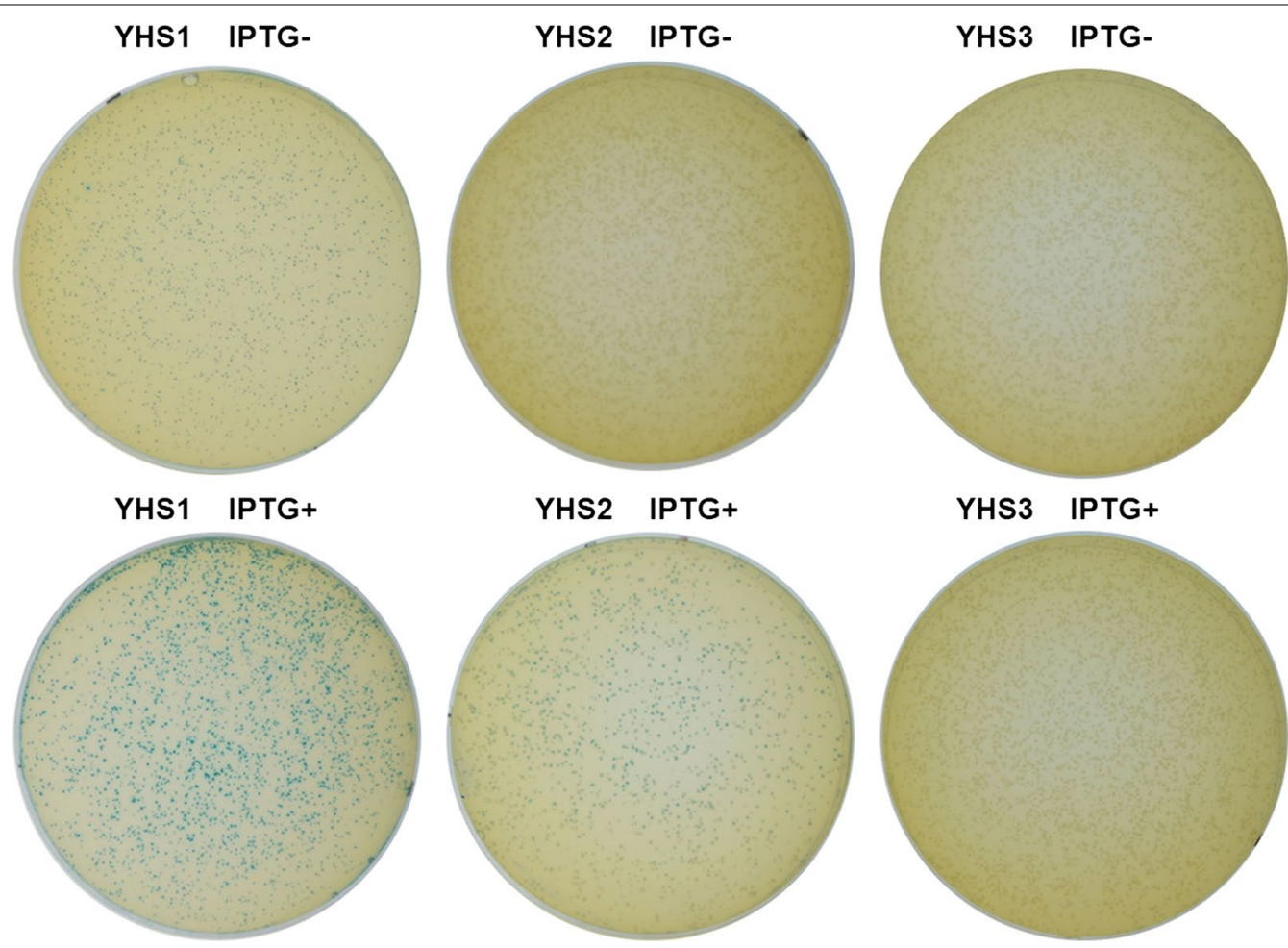

Fig. 3 Optimization of copy number of the plasmid for BmoR- $P_{b m o}$ device construction via blue-white screening experiments. The $B m o R-P_{b m o}$ device in the strain YHS1 (BW25113 (F') harboring PSA69 and pYH4) was constructed in a high-copy-number plasmid. The BmoR- $P_{b m o}$ device in the strain YHS2 (BW25113 (F') harboring pSA69, pSA65 and pYH5) was constructed in a medium-copy-number plasmid. The BmoR- $P_{b m o}$ device in the strain YHS3 (BW25113 (F') harboring pSA69, pSA65 and pYH3) was constructed in a low-copy-number plasmid 
Subsequently, the BmoR- $P_{b m o}$ device in medium-copynumber plasmid was used for the following experiments.

\section{Establishment of the screening system}

For conducting the subsequent screening experiment, we firstly established a screening system based on bluewhite screening. Blue-white screening experiment was carried out in strain YHS4 which did not contain pSA69, an enhancing precursor production plasmid and the results showed the colonies were able to turn blue though the strain was wild-type (data not shown). Based on this result, we concluded the blue-white screening was not suitable for screening the high-level isobutanol production strains from the ARTP mutant library. In this study, we also constructed a GFP fluorescence-based screening system. Plasmid pSA65 containing kivd and adhA genes which were under the control of an IPTG-inducible promoter $P_{L} l a c O_{1}$ and used for conversion of 2-ketoisovalerate to isobutanol and plasmid pYH10 containing BmoR- $P_{b m o}$ device were introduced into E. coli BW25113 $\left(\mathrm{F}^{\prime}\right)$ together to obtain strain YHS5. As the results shown in Fig. 4, the GFP/OD 600 value was increased consistently throughout the fermentation process $(48 \mathrm{~h}$ ), which was in sync with the raise of isobutanol titer. The strain reached a highest $\mathrm{GFP} / \mathrm{OD}_{600}$ value $(252.6 \pm 13.2)$ at $48 \mathrm{~h}$, while $797.3 \pm 103.1 \mathrm{mg} / \mathrm{L}$ isobutanol was accumulated during the same period. These results suggested that this GFP fluorescence-based system could be applied for the following screening experiments.

\section{Screening high-level isobutanol production strains}

A mutant library of E. coli BW25113 $\left(\mathrm{F}^{\prime}\right)$ was obtained by using the ARTP method. The mutated cells were

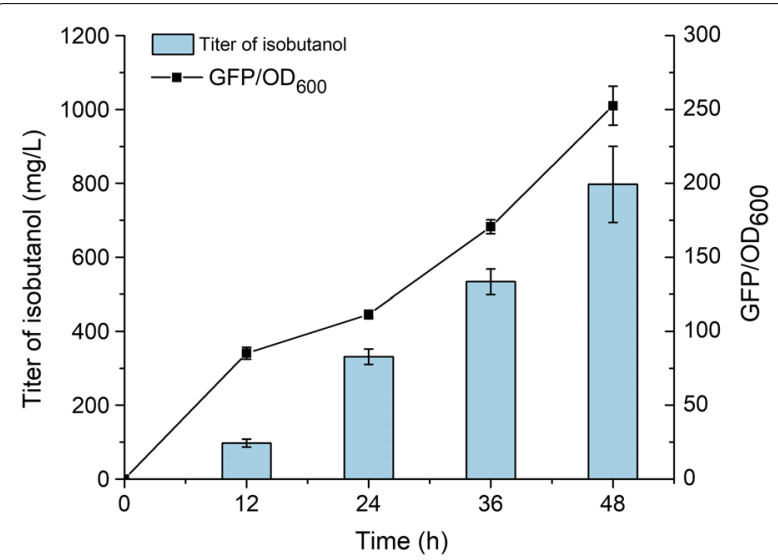

Fig. 4 Verification of the relevance between isobutanol production and GFP/OD 600 value of the host strain. For this experiment, strain YHS5 (BW25113 (F') harboring pSA65 and PYH10) which have the BmoR-based biosensor and the ability to produce isobutanol was used co-transformed with plasmids pYH10 and isobutanolproducing plasmid pSA65, and 200 single colonies of these engineered strains were inoculated into M9 medium individually after pre-incubation in LB. The M9 medium was without yeast extract because the $\mathrm{L}$-valine in yeast extract could be as precursor for isobutanol production which might interfere the screening process. After measuring GFP/OD 600 value of every colony, we screened out three strains, mutant 9,10 and 30, which exhibited GFP/OD 600 values of $178.2 \pm 15.6,190.7 \pm 4.8$ and $170.5 \pm 1.9$, respectively (Fig. $5 \mathrm{a}$ ). The GFP/OD 600 values of mutant 9,10 and 30 were 1.3, 1.4 and 1.3-fold higher GFP/OD 600 values than that of wild type (YHS5), respectively. According to these results, we speculated that these three mutants might be able to produce more isobutanol than wild type in the absence of overexpression of upstream pathway for precursor 2-KIV production. Subsequently, shake flask experiment was adopted to test the isobutanol production of these three mutated strains. The growth curves of the mutants were shown in Fig. 5b. The trend of the growth curves of mutant 9, 10 and 30 were similar to that of wild type and the cell growths were increased continuously within $48 \mathrm{~h}$. Significantly, the $\mathrm{OD}_{600}$ values of mutant 9,10 and 30 were lower than that of wild type during the whole fermentation process. Mutant 30 had an $\mathrm{OD}_{600}$ value of $0.5 \pm 0.02$ at $48 \mathrm{~h}$, a 1.7 -fold lower value than that of wild type at the same time point. The $\mathrm{OD}_{600}$ value of mutant 10 at $48 \mathrm{~h}$ was $0.7 \pm 0.1$ which was close to that of wild type. These results suggested that the mutagenesis affected the growth of E. coli BW25113 (F'). Fig 5c, d showed that the GFP/OD 600 values and the isobutanol productions of wild type and mutants during the fermentation, respectively. The GFP/OD 600 values were increased with the isobutanol titer. The producing of isobutanol in these strains was continuous during the whole fermentation process and the isobutanol titer attained the highest value at $48 \mathrm{~h}$. As expected, all the mutants could produce more isobutanol than wild type at any point in the fermentation process, which corresponding to their GFP/ $\mathrm{OD}_{600}$ value. Within $48 \mathrm{~h}, 1506.7 \pm 99.3,1597.6 \pm 129.6$ and $1436.8 \pm 157.7 \mathrm{mg} / \mathrm{L}$ isobutanol were accumulated in the cultures of mutant 9, 10 and 30, respectively. Remarkably, the isobutanol titer of mutant 10 was highest among these three mutants, representing a 2.0 -fold higher value when compared with that of wild type. These results demonstrated that BmoR- $P_{b m o}$ device could be used as an efficient tool to screen high-level isobutanol-producing strains via measuring GFP fluorescence. 


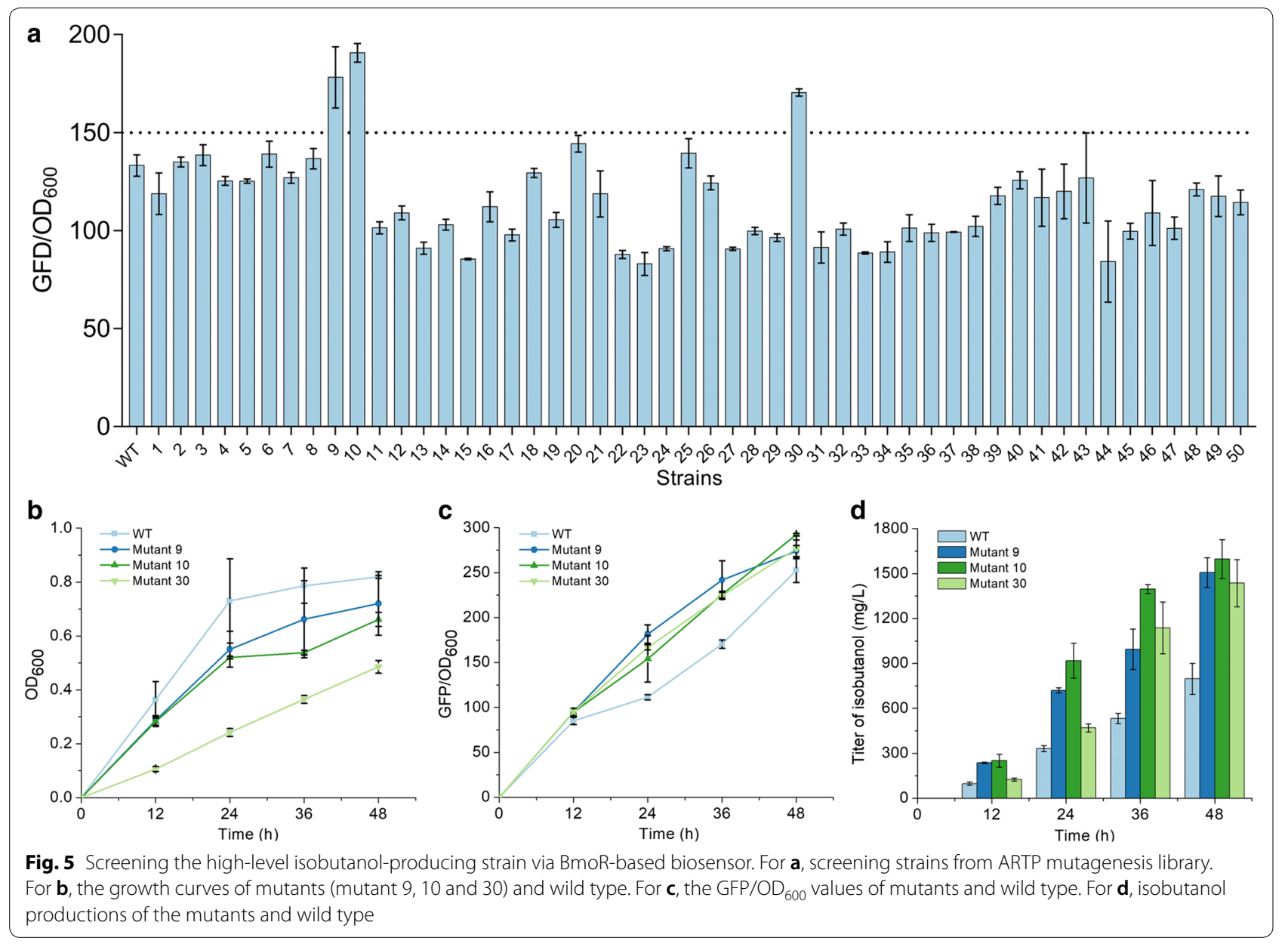

\section{Improvement of isobutanol production via strengthening the upstream pathway and optimizing yeast extract concentration}

Based on the above results, mutant 10 was selected as a candidate to achieve the overproduction of isobutanol in shake flask. Firstly, to enhance the supply of precursor (2-KIV), plasmid pSA69 was introduced into mutant 10 (strain YHS6) to express AlsS, IlvC and IlvD which included in the 2-ketoisovalerate biosynthetic pathway. High concentration of yeast extract was beneficial for heterologous protein expressions, which might improve the desired chemical production. On the other hand, the fast cell growth facilitated by the high amount of yeast extract would consume more carbon sources, leading to the decrease of desired compound production. Therefore, optimization of yeast extract concentration was an essential step for enhancing the isobutanol production. M9 medium with $40 \mathrm{~g} / \mathrm{L}$ glucose and different concentrations of yeast extract $(0,1,2,3,4$ or $5 \mathrm{~g} / \mathrm{L})$ was used for the fermentation of strain YHS6. As the results shown in Fig. 6, the isobutanol titer increased along the time course regardless of the yeast extract concentration.
Besides, the production of isobutanol was improved continuously with the raise of yeast extract concentration until the yeast extract concentration reached $4 \mathrm{~g} / \mathrm{L}$.

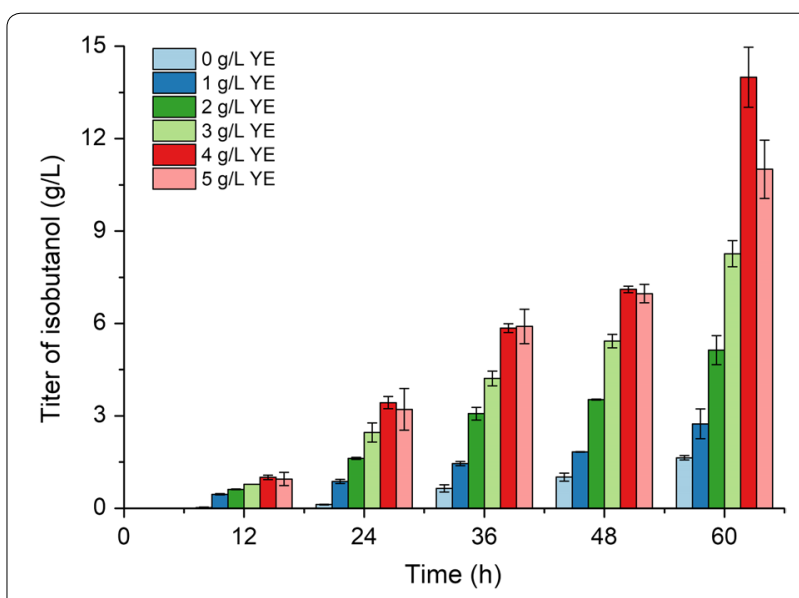

Fig. 6 Optimization of yeast extract concentration for isobutanol production. For this experiment, strain YHS6 (mutant 10 harboring plasmid pSA69) was used 
With $4 \mathrm{~g} / \mathrm{L}$ yeast extract adding into the medium, the isobutanol titer attained $14.0 \pm 1.0 \mathrm{~g} / \mathrm{L}$ at $60 \mathrm{~h}$, which was 8.8-fold higher than that of mutant 10 without optimization. Further increasing yeast extract concentration to $5 \mathrm{~g} / \mathrm{L}$ did not enhance the isobutanol production. These results suggested that reinforcing the upstream pathway and optimizing yeast extract concentration was effective to improve isobutanol biosynthesis.

\section{Production of isobutanol in a fed-batch bioreactor}

A 3-L bioreactor was employed to examine the potential of the isobutanol production capability of mutant 10 . To conduct this experiment, the strain YHS6 (mutant 10 harboring plasmid pSA69) was used. The schematic diagram of the fed-batch fermentation equipment was shown in Fig. 7. The isobutanol which yielded by the YHS6 in the broth would be stripped out and condensed by the condenser, and then was collected into the bottle $\mathrm{A}, \mathrm{B}$ and $\mathrm{C}$. The results of fed-batch culture were shown in Fig. 8. Figure 8a displayed the total isobutanol production which was calculated as the sum of isobutanol in collection bottle A, B, C and the broth with a working volume of $2 \mathrm{~L}$, and the isobutanol production in the broth. In the first $12 \mathrm{~h}$ after induction, the isobutanol was produced continuously and the titer reached $2.9 \pm 0.3 \mathrm{~g} / \mathrm{L}$ in the broth at $12 \mathrm{~h}$. At the same time point, the total of isobutanol production was $3.7 \pm 0.3 \mathrm{~g} / \mathrm{L}$, suggesting that the large proportion of isobutanol was remained in the broth which might inhibit the growth of the cells. Based on that, the air flow rate was raised to $3 \mathrm{vvm}$ (the abbreviation of air volume/culture volume/min) in order to remove isobutanol from the broth. In 20-52 h, the isobutanol titer in the broth was maintained less than $3 \mathrm{~g} / \mathrm{L}$. In the subsequent fermentation (56-82 h), the isobutanol titer in the broth was increased and reached $4.7 \pm 0.6 \mathrm{~g} / \mathrm{L}$ at $82 \mathrm{~h}$. At the end of the fermentation (106 h), the isobutanol titer in the broth was only $1.3 \pm 0.1 \mathrm{~g} / \mathrm{L}$ and the total isobutanol titer reached $56.5 \pm 1.8 \mathrm{~g} / \mathrm{L}$, which was 4.0-fold higher than that of shake flask experiment. In the previous reported study, the wild-type strain $E$. coli BW25113 $\left(\mathrm{F}^{\prime}\right)$ containing isobutanol production pathway could accumulate $41.4 \pm 4.5 \mathrm{~g} / \mathrm{L}$ isobutanol in $72 \mathrm{~h}$ in a 1-L bioreactor [35]. Compared with the above reported study, the isobutanol titer in this present study was 1.4fold higher than that of E. coli BW25113 $\left(\mathrm{F}^{\prime}\right)$. This result illustrated that we used the BmoR-based biosensor to screen out a promising strain which could be applied for high-level isobutanol production. Fig $8 \mathrm{~b}$ showed the biomass and glucose concentration at different time point in the whole fermentation process. The strain YHS6 reached the maximum cell density at $56 \mathrm{~h}$. After this time point, the cells stopped growing and the cell density was

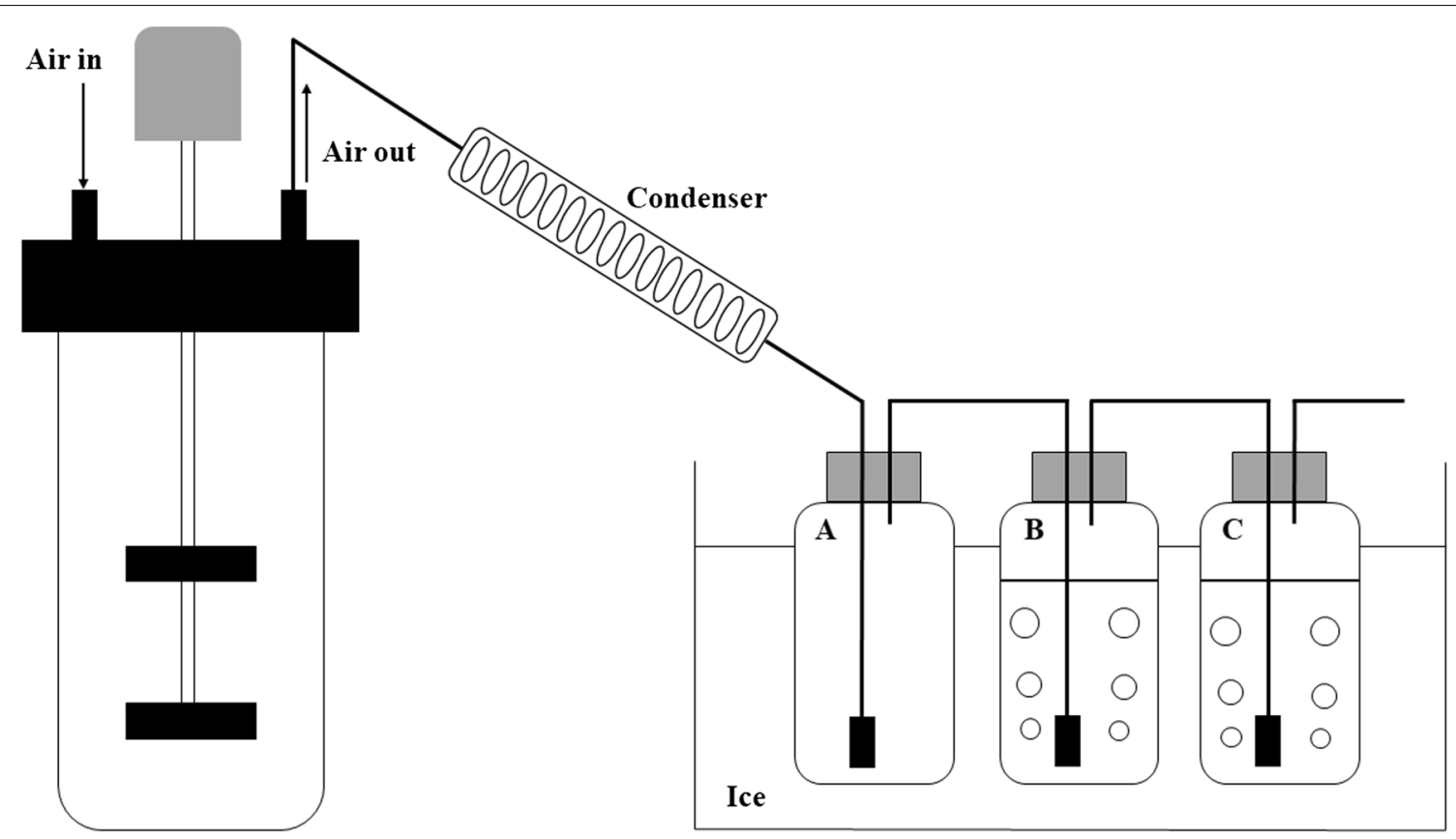

Bioreactor

Fig. 7 Schematic diagram of the fed-batch fermentation equipment for isobutanol production by strain YHS6 (mutant 10 harboring plasmid pSA69). Bottle A was empty and cooled with ice to collect the condensed isobutanol. Bottles B and C containing $800 \mathrm{~mL}$ water was also cooled with ice to collect the residual uncondensed isobutanol 

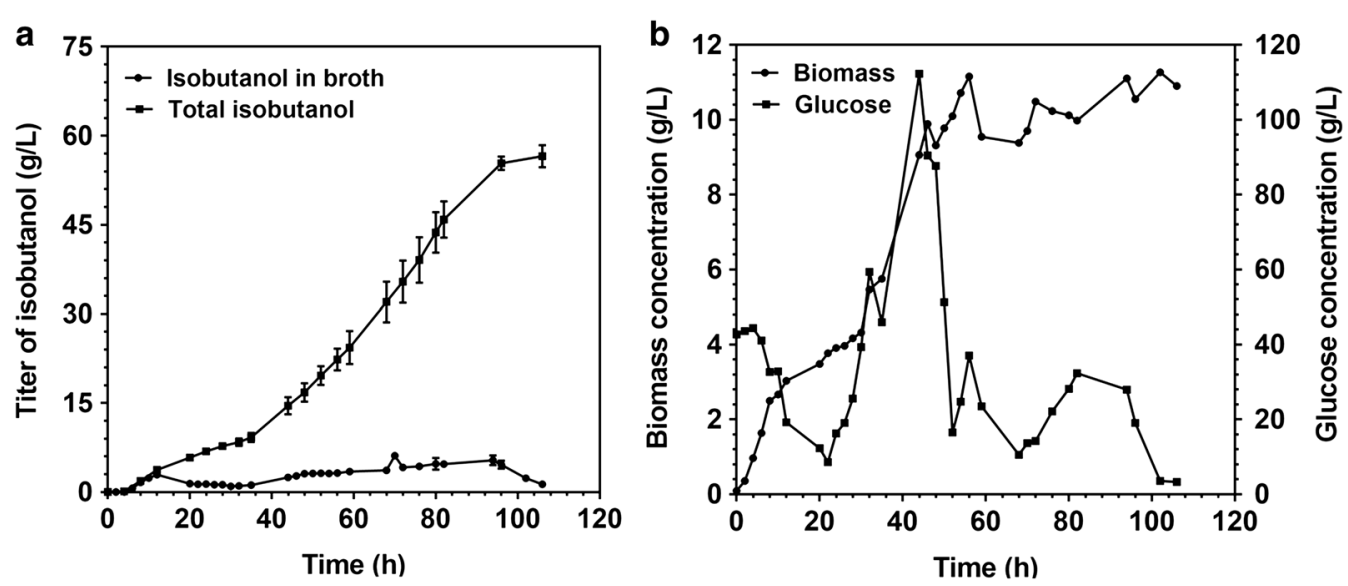

Fig. 8 Typical kinetics of isobutanol production by strain YHS6 (mutant 10 harboring plasmid pSA69) in a 3-L bioreactor at $30^{\circ} \mathrm{C}$. For a, total isobutanol production which was calculated as sum of isobutanol in collection bottles A, B, C and the broth with a working volume of $2 \mathrm{~L}$, and the isobutanol production in broth. For $\mathbf{b}$, cell growth and glucose consumption during the whole fermentation process

maintained at a relatively constant value. During the fermentation process, the stock solution containing $500 \mathrm{~g} / \mathrm{L}$ glucose was fed to the culture to maintain the glucose concentration in broth above $5 \mathrm{~g} / \mathrm{L}$ because the glucose was the main substrate for isobutanol production [8] and the low glucose concentration for a long time would result in the decrease of the isobutanol production.

\section{Conclusions}

In this work, we confirmed that the biosensor BmoR could respond to isobutanol and could be utilized as a basic element for screening isobutanol-overproducing strains. We assembled a BmoR- $P_{\text {bmo }}$ system and then combined it with isobutanol biosynthetic pathway to construct a GFP-based screening strategy in E. coli. From an ARTP mutagenesis library, we screened out three strains which have ability to produce more isobutanol than wild type in the absence of overexpression of upstream pathway for precursor 2-KIV production. Besides, reinforcing the 2-KIV overproduction pathway and optimizing medium improved the isobutanol titer from a mutated host to $14.0 \pm 1.0 \mathrm{~g} / \mathrm{L}$. Moreover, the titer of isobutanol reached $56.5 \pm 1.8 \mathrm{~g} / \mathrm{L}$ in a fed-batch production experiment.

\section{Methods}

\section{Medium, strains and plasmids}

$\mathrm{LB}(10 \mathrm{~g} / \mathrm{L}$ tryptone, $5 \mathrm{~g} / \mathrm{L}$ yeast extract and $10 \mathrm{~g} / \mathrm{L} \mathrm{NaCl})$ medium was used for cell incubation and plasmid construction. M9 medium which contains $6 \mathrm{~g} / \mathrm{L} \mathrm{Na}_{2} \mathrm{HPO}_{4}$, $3 \mathrm{~g} / \mathrm{L} \mathrm{KH}_{2} \mathrm{PO}_{4}, 1 \mathrm{~g} / \mathrm{L} \mathrm{NH} \mathrm{NH}_{4} \mathrm{Cl}$ and $0.5 \mathrm{~g} / \mathrm{L} \mathrm{NaCl}, 1 \mathrm{M}$ $\mathrm{MgSO}_{4}, 0.1 \mathrm{M} \mathrm{CaCl}_{2}, 10 \mathrm{mg} / \mathrm{L}$ vitamin $\mathrm{B} 1$ and $20 \mathrm{~g} / \mathrm{L}$ glucose was used for mutagenesis screening and fermentation confirmation. For yeast concentration optimization, glucose concentration in M9 medium was changed to $40 \mathrm{~g} / \mathrm{L}$ and various concentration of yeast extract $(0,1$, $2,3,4$ or $5 \mathrm{~g} / \mathrm{L}$ ) was added in the medium. E. coli XL1Blue was used for plasmid construction, while E. coli BW25113 $\left(\mathrm{F}^{\prime}\right)$ was used for feeding experiments and as parent strain for ARTP mutagenesis. The details of strains and plasmids were described in Table 2.

\section{DNA manipulation}

Plasmids pUC19 from TransGen Biotech, pYK [16] and pSA74 [36] were used as templates to amplify genes lac $Z \alpha$, colA origin and $p S C 101 *$ origin, respectively. Plasmids pSA65 and pSA69 were from previous study [8]. Plasmid pSA65 was used as template to amplify colE1 origin. Gene $g f p$ (accession number: AAX07425.1) was synthesized by OE-PCR. The $P_{b m o R}-b m o R$ and $P_{b m o}$ were referenced from NCBI (accession number: AY093933.3). $P_{b m o R}-b m o R$ was synthesized by OE-PCR after codon optimization for E. coli and then ligated with colE1 origin, $a m p^{r}$ and $P_{b m o}-g f p$ via Gibson Assembly, generating plasmid pYH1. To construct plasmid pYH2, $g f p$ of pYH1 was replaced with $l a c Z \alpha$. Replacing colE1 origin and $a m p^{r}$ of pYH2 with $p S C 101 *$ origin and $\mathrm{cm}^{r}$, respectively, generated plasmid pYH3. To create plasmid pYH5, pSC101* origin of pYH3 was substituted with colA origin. $P_{b m o R^{-}}$ bmoR, $P_{\text {bmo }}$-lac $Z \alpha, P_{L} l a c O_{1}$-kivd-adhA which was amplified from pSA65, colE1 origin and $a m p^{r}$ were assembled via Gibson Assembly to generate plasmid pYH4. Gene $l a c Z \alpha$ of pYH3, pYH4 and pYH5 were replaced with $g f p$, resulting in plasmids pYH11, pYH9 and pYH10, respectively. All the plasmids were sequenced by GENEWIZ company. 
Table 2 Plasmids and strains used in this study

\begin{tabular}{|c|c|c|}
\hline & Description & Source \\
\hline \multicolumn{3}{|l|}{ Plasmids } \\
\hline pUC19 & $P_{\text {lac }}-\mid a c Z a ; c o l E 1 ; a m p^{r}$ & TransGen Biotech \\
\hline pYK & $P_{L} \mathrm{lacO}_{1} ; \mathrm{LaCl}^{\prime} \mathrm{col} ; \mathrm{kan}^{r}$ & {$[16]$} \\
\hline pSA74 & $P_{L} l a c O_{1} ; p C 101^{*} ; \mathrm{cm}^{r}$ & {$[36]$} \\
\hline pSA65 & $P_{L} l a c O_{1}$-kivd-adhA; colE1; ampr & {$[8]$} \\
\hline pSA69 & $P_{L} l a c O_{1}$-alsS-ilvC-ilvD; p15A; $\mathrm{kan}^{r}$ & {$[8]$} \\
\hline pYH1 & $P_{b m o R}-b m o R ; P_{b m o}-g f p ; c o l E 1 ; a m p^{r}$ & This study \\
\hline pYH2 & $P_{b m o R}-b m o R ; P_{b m o}-l a c Z a ; c o l E 1 ; a m p^{r}$ & This study \\
\hline pYH3 & $P_{b m o R^{-}}{ }^{-b m o R ;} P_{b m o}-l a c Z a ; p S C 101^{*} ; \mathrm{cm}^{r}$ & This study \\
\hline pYH4 & $P_{b m o R}-b m o R ; P_{b m o}-l a c Z a ; P_{L} l a c O_{1}-$-kivd-adhA; colE $1 ; a m p^{r}$ & This study \\
\hline pYH5 & $P_{b m o R}-b m o R ; P_{b m o}-l a c Z a ; c o l A ; \mathrm{cm}^{r}$ & This study \\
\hline pYH9 & $P_{b m o R}-b m o R ; P_{b m o}-g f p ; P_{L} l a c O_{1}-k i v d-a d h A ; c o l E 1 ; a m p^{r}$ & This study \\
\hline pYH10 & $P_{b m o R}-b m o R ; P_{b m o}-g f p ; c o l A ; \mathrm{cm}^{r}$ & This study \\
\hline pYH11 & 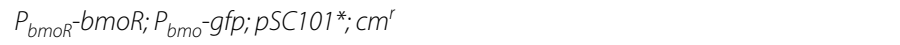 & This study \\
\hline \multicolumn{3}{|l|}{ Strains } \\
\hline XL1-Blue & recA1 endA1 gyrA96 thi-1 hsdR17 supE44 relA1 lac [F' proAB lac $1^{9} Z \Delta M 15 \operatorname{Tn} 10\left(\right.$ Tet $\left.\left.^{\prime}\right)\right]$ & {$[8]$} \\
\hline BW25113 (F') & 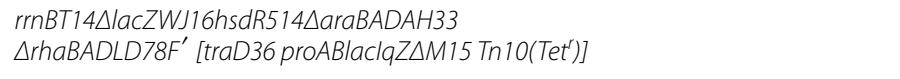 & {$[8]$} \\
\hline $\mathrm{YH} 1$ & BW25113 (F') with pYH1 & This study \\
\hline $\mathrm{YH} 2$ & BW25113 (F') with pYH2 & This study \\
\hline YHS1 & BW25113 (F') with pSA69 and pYH4 & This study \\
\hline YHS2 & BW25113 (F') with pSA69, pSA65 and pYH5 & This study \\
\hline YHS3 & BW25113 (F') with pSA69, pSA65 and pYH3 & This study \\
\hline YHS4 & BW25113 (F') with pSA65 and pYH5 & This study \\
\hline YHS5 & BW25113 (F') with pSA65 and pYH10 & This study \\
\hline YHS6 & Mutant 10 with pSA69 & This study \\
\hline
\end{tabular}

\section{Extracellular confirmation of BmoR- $P_{b m o}$ biosensor based on GFP fluorescence}

Escherichia coli BW25113 (F') harboring plasmid pYH1 was pre-inoculated into $5 \mathrm{~mL} \mathrm{LB}$ with $100 \mu \mathrm{g} / \mathrm{mL}$ ampicillin and then cultured at $37{ }^{\circ} \mathrm{C}$ overnight. Then, $50 \mu \mathrm{L}$ of the seed culture was transferred into $950 \mu \mathrm{L}$ fresh LB medium supplemented with different concentrations $(0$, $0.01,0.1,1,10,20,40,50$ or $100 \mathrm{mM}$ ) of $n$-butanol, isobutanol and 3-MB, respectively, in 96-deep-well plates and the cultures were then left at $30{ }^{\circ} \mathrm{C}$ for $16 \mathrm{~h}$. After that, GFP fluorescence and $\mathrm{OD}_{600}$ values were detected by microplate reader (BioTek Cytation 3). GFP fluorescence was measured using an excitation wavelength of $470 \mathrm{~nm}$ and an emission wavelength of $510 \mathrm{~nm}$. GFP fluorescence value was normalized as GFP/OD 600 , and the background fluorescence of medium was subtracted. $K_{\mathrm{m}}$ values were estimated with OriginPro8.5 through non-linear regression of the Hill1 equation.
Extracellular confirmation of BmoR- $P_{b m o}$ biosensor based on blue-white screening

Escherichia coli BW25113 (F') harboring the biosensor plasmid pYH2 were cultured overnight in $5 \mathrm{~mL} \mathrm{LB}$ medium with $100 \mu \mathrm{g} / \mathrm{mL}$ ampicillin, and then $50 \mu \mathrm{L}$ of seed culture was transferred into $5 \mathrm{~mL}$ fresh LB medium with different concentrations of isobutanol $(0,0.01,0.1$, $1,10,20,40,50$ or $100 \mathrm{mM}$ ) feeding when $\mathrm{OD}_{600}$ reached 0.2-0.4. Cultures were then left at $30{ }^{\circ} \mathrm{C}$ for $24 \mathrm{~h}$, and then $10 \mu \mathrm{L}$ of the seed culture was diluted 5000 times and spread on the LB plate with $100 \mu \mathrm{g} / \mathrm{mL}$ X-gal, different concentrations of isobutanol and associated antibiotics. The plates were placed in a $30{ }^{\circ} \mathrm{C}$ incubator for $72 \mathrm{~h}$.

\section{Verifying BmoR- $P_{b m o}$ biosensor response to isobutanol produced by cell based on blue-white screening} Plasmids with different copies (pYH4, pYH5 and pYH3, high-copy, medium-copy and low-copy-number, respectively) were introduced into isobutanol-producing strain E. coli BW25113 (F') (pSA65/pSA69). The single colonies were inoculated into $5 \mathrm{~mL} \mathrm{LB}$ medium with appropriate antibiotics and grew at $37{ }^{\circ} \mathrm{C}$ until $\mathrm{OD}_{600}$ reached around 
1.0. Then, $10 \mu \mathrm{L}$ of the seed culture was diluted 5000 times and spread on the M9 plate with $100 \mu \mathrm{g} / \mathrm{mL} X$-gal, $0.1 \mathrm{mM}$ IPTG and associated antibiotics. The plates were placed in a $30{ }^{\circ} \mathrm{C}$ incubator for $72 \mathrm{~h}$.

\section{ARTP mutagenesis and screening}

The ARTP mutation system which could cause greater gene damage than traditional mutagenesis was employed to generate the mutation library [37]. E. coli BW25113 $\left(\mathrm{F}^{\prime}\right)$, as initial strain, was pre-incubated into $5 \mathrm{~mL} \mathrm{LB}$ medium and cultured at $37^{\circ} \mathrm{C}$ for $4 \mathrm{~h}$ to reach its logarithmic phase. Then, $10 \mu \mathrm{L}$ of the culture was transferred to stainless steel minidisc and subsequently exposed to ARTP with 60-s irradiation. The mutated cells were recovered in $3 \mathrm{~mL}$ fresh $\mathrm{LB}$ medium at $37^{\circ} \mathrm{C}$ for $6 \mathrm{~h}$ and then stored in $15 \%$ glycerol for screening. For screening, the cells in mutagenesis library were transformed with plasmids pSA65 and pYH10. The single colonies were pre-incubated into $5 \mathrm{~mL} \mathrm{LB}$ medium with $100 \mu \mathrm{g} /$ $\mathrm{mL}$ ampicillin and $25 \mu \mathrm{g} / \mathrm{mL}$ chloromycetin at $37{ }^{\circ} \mathrm{C}$ for $12 \mathrm{~h} .50 \mu \mathrm{L}$ of the seed culture was added into $1 \mathrm{~mL}$ M9 medium with $0.1 \mathrm{mM}$ IPTG in 96-deep-well plates. All 96-deep-well plates with cultures were incubated at $30^{\circ} \mathrm{C}$ for $20 \mathrm{~h}$. The fluorescence and $\mathrm{OD}_{600}$ values were measured by the microplate reader.

\section{Fermentation verification of the isobutanol overproduction strains in shake flask}

The single colonies were pre-inoculated into $5 \mathrm{~mL} \mathrm{LB}$ medium with associated antibiotics at $37{ }^{\circ} \mathrm{C}$ for $12 \mathrm{~h}$. Then, $200 \mu \mathrm{L}$ culture was inoculated into $20 \mathrm{~mL}$ M9 with $0.1 \mathrm{mM}$ IPTG and various concentration of yeast extract in $250 \mathrm{~mL}$ screw cap conical flask and then left at $30^{\circ} \mathrm{C}$. Samples were taken every $12 \mathrm{~h}$. Then, $200 \mu \mathrm{L}$ of sample was added into 96-well plates for fluorescence and $\mathrm{OD}_{600}$ measurement. Production of alcohols was quantified by Agilent $6890 \mathrm{GC}$ chromatograph equipped with flame ionization detector (Agilent Technologies, CA, USA). The separation of alcohols was carried out by a DB-FFAP capillary column $(30 \mathrm{~m} \times 0.32 \mathrm{~mm} \times 0.25 \mu \mathrm{m}$; Agilent Technologies). For analysis of isobutanol, the GC oven temperature was initially held at $80{ }^{\circ} \mathrm{C}$ for $3 \mathrm{~min}$, increased with a gradient of $115{ }^{\circ} \mathrm{C} / \mathrm{min}$ until $230{ }^{\circ} \mathrm{C}$, and kept at $230{ }^{\circ} \mathrm{C}$ for an additional $1 \mathrm{~min}$. Nitrogen was used as the carrier gas. The injector and detector were maintained at 250 and $280{ }^{\circ} \mathrm{C}$, respectively. Supernatant (1 $\mu \mathrm{L})$ was sampled and injected at a split ratio of 1:30 and $\mathrm{n}$-pentanol was used as internal standard.

\section{Culture medium for fed-batch fermentation}

To evaluate the isobutanol-producing potential of the mutated strain which was screened out from the mutation library, the fermentation was performed in a
3-L bioreactor with $2 \mathrm{~L}$ working volume. The culture medium containing $40 \mathrm{~g} / \mathrm{L}$ glucose, $3 \mathrm{~g} / \mathrm{L}\left(\mathrm{NH}_{4}\right)_{2} \mathrm{SO}_{4}$, $14.6 \mathrm{~g} / \mathrm{L} \mathrm{K}_{2} \mathrm{HPO}_{4}, 4 \mathrm{~g} / \mathrm{L} \mathrm{KH}_{2} \mathrm{PO}_{4}, 2.2 \mathrm{~g} / \mathrm{L}$ sodium citrate, $8 \mathrm{~g} / \mathrm{L}$ yeast extract, $1.25 \mathrm{~g} / \mathrm{L} \mathrm{MgSO}_{4} \cdot 7 \mathrm{H}_{2} \mathrm{O}, 0.1 \mathrm{~g} / \mathrm{L}$ ampicillin, $0.05 \mathrm{~g} / \mathrm{L}$ kanamycin, $0.025 \mathrm{~g} / \mathrm{L}$ chloromycetin and $1 \mathrm{~mL} / \mathrm{L}$ trace metal solution was used for bioreactor fermentation. Trace metal solution contained 14.1 g EDTA, $2.5 \mathrm{~g} \mathrm{CoCl}_{2} \cdot 6 \mathrm{H}_{2} \mathrm{O}, 15 \mathrm{~g} \mathrm{MnCl}_{2} \cdot 4 \mathrm{H}_{2} \mathrm{O}, 1.5 \mathrm{~g}$ $\mathrm{CuCl}_{2} \cdot 2 \mathrm{H}_{2} \mathrm{O}, 3 \mathrm{~g} \mathrm{H}_{3} \mathrm{BO}_{3}, 2.1 \mathrm{~g} \mathrm{Na}_{2} \mathrm{MoO}_{4} \cdot 2 \mathrm{H}_{2} \mathrm{O}, 33.8 \mathrm{~g}$ $\mathrm{Zn}\left(\mathrm{CH}_{3} \mathrm{COO}\right)_{2} \cdot 2 \mathrm{H}_{2} \mathrm{O}$ and $80 \mathrm{~g} \mathrm{FeCl}_{3} \cdot 6 \mathrm{H}_{2} \mathrm{O}$ per liter. During the cultivation period, $1.5 \mathrm{~L}$ stock solution containing $500 \mathrm{~g} / \mathrm{L}$ glucose, $1.25 \mathrm{~g} / \mathrm{L} \mathrm{MgSO}_{4} \cdot 7 \mathrm{H}_{2} \mathrm{O}, 0.1 \mathrm{~g} / \mathrm{L}$ ampicillin, $0.05 \mathrm{~g} / \mathrm{L}$ kanamycin, $0.025 \mathrm{~g} / \mathrm{L}$ chloromycetin and $0.1 \mathrm{mM}$ IPTG was fed to the batch culture.

\section{Bioreactor culture conditions}

The bioreactor was inoculated with $5 \%$ of overnight preculture and the cells grown at $37{ }^{\circ} \mathrm{C}$ with 1 vvm of air flow rate and $600 \mathrm{rpm}$ of stirrer speed for $2 \mathrm{~h}$. Then, $0.1 \mathrm{mM}$ IPTG was added into the bioreactor and the temperature was changed to $30{ }^{\circ} \mathrm{C}$ to induce the expression of the enzymes in isobutanol production pathway. The $\mathrm{pH}$ was controlled at 6.8 by automatic addition of ammonia solution (25\%). After induction of $12 \mathrm{~h}$, the air flow rate was increased from 1 to $3 \mathrm{vvm}$ in order to strip out isobutanol from the broth. The evaporated isobutanol was condensed by a condenser and subsequently the generated liquid isobutanol flowed into collection bottle A which was cooled with ice (Fig. 7). The residual uncondensed isobutanol was collected into bottle $\mathrm{B}$ and $\mathrm{C}$ which containing $800 \mathrm{~mL}$ water and were also cooled with ice (Fig. 7). The samples were taken to determinate the biomass, glucose concentration and isobutanol titer.

\section{Authors' contributions}

YH generated the idea. YH, ZC and HY designed the project. HY, ZC, NW and $\mathrm{WH}$ conducted the experiments. $Y Z$ and $W Z$ directed the fed-batch fermentation experiments. YH, YY, HY and ZC analyzed the data. YH, HY and ZC wrote the manuscript. All authors read and approved the final manuscript.

\section{Author details}

${ }^{1}$ Key Laboratory of Molecular Medicine and Biotherapy, School of Life Science, Beijing Institute of Technology, No. 5 South Zhongguancun Street, Beijing 100081, China. ${ }^{2}$ Biotechnology Research Institute of Chinese Academy of Agricultural Sciences, No. 12 South Zhongguancun Street, Beijing 100081, China.

\section{Acknowledgements}

The authors wish to thank Beijing Institute of Technology for the supply of microplate reader (BioTek Cytation 3) and Biotechnology Research Institute of Chinese Academy of Agricultural Sciences for the supply of fluorescence microscope (Nikon model Eclipse $\mathrm{Ni}-\mathrm{U}$ ) and bioreactors (Bioengineering $\mathrm{AG}$ ).

\section{Competing interests}

The authors declare that they have no competing interests. 


\section{Availability of data and materials}

All the data generated during the current study are included in the manuscript.

\section{Consent for publication \\ Not applicable.}

\section{Ethics approval and consent to participate}

Not applicable.

\section{Funding}

The authors would like to acknowledge financial support of the National Natural Science Foundation of China (Grant No. 21676026), the China Postdoctoral Science Foundation Funded Project (Grant No. 2018M630083) and Fundamental Research Funds for the Central Universities.

\section{Publisher's Note}

Springer Nature remains neutral with regard to jurisdictional claims in published maps and institutional affiliations.

Received: 8 October 2018 Accepted: 31 January 2019

Published online: 07 February 2019

\section{References}

1. Connor MR, Liao J. Microbial production of advanced transportation fuels in non-natural hosts. Curr Opin Biotechnol. 2009;20:307-15.

2. Lütke-Eversloh T, Stephanopoulos G. Combinatorial pathway analysis for improved L-tyrosine production in Escherichia coli: identification of enzymatic bottlenecks by systematic gene overexpression. Metab Eng. 2008;10:69-77.

3. Avalos JL, Fink GR, Stephanopoulos G. Compartmentalization of metabolic pathways in yeast mitochondria improves production of branched chain alcohols. Nat Biotechnol. 2013:31:335-43.

4. Ro DKPE, Ouellet M, Fisher KJ, Newman KL, Ndungu JM, Ho KA, Eachus RA, Ham TS, Kirby J, Chang MC, Withers ST, Shiba Y, Sarpong R, Keasling JD. Production of the antimalarial drug precursor artemisinic acid in engineered yeast. Nature. 2006;440:940-3.

5. Park SH, Kim HU, Kim TY, Park JS, Kim SS, Lee SY. Metabolic engineering of Corynebacterium glutamicum for L-arginine production. Nat Commun. 2014;5:4618.

6. Chen Z, Sun X, Li Y, Yan Y, Yuan Q. Metabolic engineering of Escherichia coli for microbial synthesis of monolignols. Metab Eng. 2016;39:102-9.

7. Chen Z, Shen X, Wang J, Wang J, Zhang R, Rey JF, Yuan Q, Yan Y. Establishing an artificial pathway for de novo biosynthesis of vanillyl alcohol in Escherichia coli. ACS Synth Biol. 2017;6:1784-92.

8. Atsumi S, Hanai T, Liao J. Non-fermentative pathways for synthesis of branched-chain higher alcohols as biofuels. Nature. 2008;451:86-9.

9. Atsumi S, Cann AF, Connor MR, Shen CR, Smith KM, Brynildsen MP, Chou KJ, Hanai T, Liao J. Metabolic engineering of Escherichia coli for 1-butanol production. Metab Eng. 2008;10:305-11.

10. Tong S, Luo Y, Han X, Zhao H. Utilizing an endogenous pathway for 1-butanol production in Saccharomyces cerevisiae. Metab Eng. 2014;22:60-8.

11. Connor MR, Liao J. Engineering of an Escherichia coli strain for the production of 3-methyl-1-butanol. Appl Microbiol Biotechnol. 2008;74:5769-75.

12. Cann AF, Liao J. Production of 2-methyl-1-butanol in engineered Escherichia coli. Appl Microbiol Biotechnol. 2008;81:89-98.

13. Li S, Wen J, Jia X. Engineering Bacillus subtilis for isobutanol production by heterologous ehrlich pathway construction and the biosynthetic 2-ketoisovalerate precursor pathway overexpression. Appl Microbiol Biotechnol. 2011;91:577-89.

14. Chen X, Nielsen KF, Borodina I, Kiellandbrandt MC, Karhumaa K. Increased isobutanol production in Saccharomyces cerevisiae by overexpression of genes in valine metabolism. Biotechnol Biofuels. 2011;4:21.

15. Smith KM, Cho KM, Liao J. Engineering Corynebacterium glutamicum for isobutanol production. Appl Microbiol Biotechnol. 2010;87:1045-55.

16. Huo Y, Cho KM, Rivera JGL, Monte E, Shen CR, Yan Y, Liao J. Conversion of proteins into biofuels by engineering nitrogen flux. Nat Biotechnol. 2011:29:346-51.
17. Adelberg EA, Mandel M, Chen G. Optimal conditions for mutagenesis by $N$-methyl-N'-nitro-N-nitrosoguanidine in Escherichia coli K12. Biochem Biophys Res Commun. 1965;18:788-95.

18. Kodym A, Afza R. Physical and chemical mutagenesis. Methods Mol Biol. 2003;236:189-204.

19. Guo T, Tang Y, Xi Y, He A, Sun B, Wu H, Liang D, Jiang M, Ouyang P. Clostridium beijerinckii mutant obtained by atmospheric pressure glow discharge producing high proportions of butanol and solvent yields. Biotechnol Lett. 2011;33:2379-83.

20. Zhang X, Zhang X, Li H, Wang L, Zhang C, Xing X, Bao C. Atmospheric and room temperature plasma (ARTP) as a new powerful mutagenesis tool. Appl Microbiol Biotechnol. 2014;98:5387-96.

21. Zhang F, Carothers JM, Keasling JD. Design of a dynamic sensor-regulator system for production of chemicals and fuels derived from fatty acids. Nat Biotechnol. 2012;30:354-9.

22. Woolston BM, Roth T, Kohale I, Liu D, Stephanopoulos G. Development of a formaldehyde biosensor with application to synthetic methylotrophy. Biotechnol Bioeng. 2018;115:206-15.

23. Siedler S, Schendzielorz G, Binder S, Eggeling L, Bringer S, Bott M. SoxR as a single-cell biosensor for NADPH-consuming enzymes in Escherichia coli. ACS Synth Biol. 2014;3:41-7.

24. Fang M, Wang T, Zhang C, Bai J, Zheng X, Zhao X, Lou C, Xing X. Intermediate-sensor assisted push-pull strategy and its application in heterologous deoxyviolacein production in Escherichia coli. Metab Eng. 2016:33:41-51.

25. Johnson AO, Gonzalez-Villanueva M, Wong L, Steinbüchel A, Tee KL, Peng $X$, Wong TS. Design and application of genetically-encoded malonyl-CoA biosensors for metabolic engineering of microbial cell factories. Metab Eng. 2017:44:253-64.

26. Liu SD, Wu YN, Wang TM, Zhang C, Xing X. Maltose utilization as a novel selection strategy for continuous evolution of microbes with enhanced metabolite production. ACS Synth Biol. 2017;6:2326-38.

27. Zhang J, Barajas JF, Burdu M, Ruegg TL, Dias B, Keasling JD. Development of a transcription factor-based lactam biosensor. ACS Synth Biol. 2016:6:439-45.

28. Skjoedt ML, Snoek T, Kildegaard KR, Arsovska D, Eichenberger M, Goedecke TJ, Rajkumar AS, Zhang J, Kristensen M, Lehka BJ. Engineering prokaryotic transcriptional activators as metabolite biosensors in yeast. Nat Chem Biol. 2016;12:951-8.

29. Schendzielorz G, Dippong M, Grünberger A, Kohlheyer D, Yoshida A, Binder S, Nishiyama C, Nishiyama M, Bott M, Eggeling L. Taking control over control: use of product sensing in single cells to remove flux control at key enzymes in biosynthesis pathways. ACS Synth Biol. 2014;3:21-9.

30. Zhang X, Zhang X, Xu G, Zhang X, Shi J, Xu Z. Integration of ARTP mutagenesis with biosensor-mediated high-throughput screening to improve L-serine yield in Corynebacterium glutamicum. Appl Microbiol Biotechnol. 2018;102:1-13.

31. Liu Y, Zhuang Y, Ding D, Xu Y, Sun J, Zhang D. Biosensor-based evolution and elucidation of a biosynthetic pathway in Escherichia coli. ACS Synth Biol. 2017;6:837-48

32. Kurth EG, Doughty DM, Bottomley PJ, Arp DJ, Sayavedrasoto LA. Involvement of BmoR and BmoG in n-alkane metabolism in 'Pseudomonas butanovora'. Microbiology. 2008;154:139-47.

33. Bush M, Dixon R. The role of bacterial enhancer binding proteins as specialized activators of $\sigma^{54}$-dependent transcription. Microbiol Mol Biol Rev. 2012;76:497-529.

34. Dietrich JA, Shis DL, Alikhani A, Keasling JD. Transcription factor-based screens and synthetic selections for microbial small-molecule biosynthesis. ACS Synth Biol. 2013;2:47-58.

35. Antonino B, Kwang-Myung C, James CL. High-flux isobutanol production using engineered Escherichia coli: a bioreactor study with in situ product removal. Appl Microbiol Biotechnol. 2011;90:1681-90.

36. Jain R, Sun X, Yuan Q, Yan Y. Systematically engineering Escherichia coli for enhanced production of 1,2-propanediol and 1-propanol. ACS Synth Biol. 2015:4:746-56.

37. Wang L, Huang Z, Li G, Zhao H, Xing X, Sun W, Li H, Gou Z, Bao C. Novel mutation breeding method for Streptomyces avermitilis using an atmospheric pressure glow discharge plasma. J Appl Microbiol. 2010;108:851-8. 